\title{
Response competition and/or inhibition in two-choice reaction time'
}

\begin{abstract}
Abstraet
A two-choice reaction time experiment was conducted in which it was demonstrated that the reaction time for a particular finger is subject to change depending on the alternatives with which it is paired. This finding, it is argued, raises questions regarding the adequacy of controls which select the experimental data from only one finger in an effort to minimize the effects of inter-finger variability. It is also a demonstration of R-R compatibility effects, and as such, lends experimental support to the hypothesis that a measurable portion of the reaction time interval is consumed by the processes associated with the inhibition of competing incorrect response alternatives.

\section{Problem}

Investigators using key pressing reaction time tasks have usually averaged times across all the fingers used in a particular condition in order to get the reaction time for that condition. However, differences in reaction time between different fingers have been noted. In fact, experiments have occasionally been designed so that all the data for the various conditions are obtained from the same finger (Hick, 1952). In this way the variability of the data attributable to differences between fingers is presumably reduced. The object of the present study is to determine whether this procedure is adequate ignoring as it does the fact that the reaction time for a particular finger is not only intrinsically different from other fingers but may also depend on the alternatives in the response repertoire being used in the experiment.

\section{Method}

A two-choice, discrete, reaction time experiment was conducted in which the stimuli were two neon lights and the responses consisted of depressing the appropriate one of two keys. In the ipsilateral condition of the experiment the response was made with either the index or the middle finger of the right hand; in the contralateral condition the response was made with either the index finger of the left hand or the middle finger of the right hand. The two lights were mounted about 1 in apart on a vertical board approximately $31 / 2 \mathrm{ft}$ in front of the subject. During the experiment extraneous distracting noises were masked by white noise which was presented to the subjects at 73-75 db through a pair of earphones. The keys required $1 / 8$ in travel and between five and six ounces pressure. Subjects were instructed to be looking at a fixation point between the two neon lights when the ready signal occurred and to keep the two relevant fingers resting on the keys at all times. A trial began with a ready signal which consisted of an interruption in the white noise lasting between 160 and $170 \mathrm{~ms}$. Two seconds after the ready signal the two stimulus lights went on. One-tenth of a second later one of the lights went off and the subject made his response to the light which had gone off. Twelve subjects participated in the experiment.

A series consisted of 48 trials. The stimuli were chosen equiprobably and randomized without replacement in each half of each series. Three series were presented on the first day, two on the second and two again on the third. The entire first day was used for training purposes. In order to give the relevant fingers some practice independent of the ipsi-contralateral order, the first series on the first day consisted of a four-choice task in which the responses were the middle and index fingers of the left and right hand. The next two series on that day were ipsilateral and contralateral with half the subjects run in one order and half in the opposite order. On the second and third day all subjects were run on an ipsilateral and contralateral series each day in a counterbalanced design.

The analysis was made for the middle finger data only. ${ }^{2}$ In order to reduce the contamination that might have occurred between series only the last half of each series was considered in the analysis. Medians were taken for each individual's performance in each series for each day. The medians for each experimental day were then combined into single means each for the ipsilateral and contralateral conditions for each individual.

\section{Results}

The mean reaction time for the ipsilateral condition was $310 \mathrm{~ms}$. while the mean reaction time for the contralateral condition was $282 \mathrm{~ms}$. Using a t-test for correlated means this $28 \mathrm{~ms}$. difference is significant at beyond the .0005 level $(t=5.19 ; d f=11)$. Of the 48 pairs of ipsilateral and contralateral series there are only three cases in which this relationship is reversed.

\section{Discussion}

Seibel (1961) and Ratz \& Ritchie (1961) in reporting reaction time to chords where the fingers in the chord are depressed simultaneously, both found that the index, middle-finger ipsilateral chord yields the second fastest reaction time among pairs. Ratz and Ritchie further report that the reaction time with contralateral chords is considerably longer than it is with ipsilateral chords. 
In taking the reaction time for individual members of such chords, however, where only one finger is depressed, the present study indicates the opposite relationship. It would, therefore, appear that the more easily a set of responses can be activated jointly the more difficult it is to activate single members of that set, and vice-versa.

These findings also provide a demonstration of $\mathrm{R}-\mathrm{R}$ compatibility effects which Fitts (1959) defines as occurring when "two responses... are carried on concurrently." In the present study, the two responses in question are the implicit preparatory or anticipatory responses. Thus, experimental suppport has also been provided for the hypothesis that a measurable portion of the reaction time interval is consumed by processes associated with the inhibition of competing incorrect alternative responses. Since the number of competing responses would increase with the number of alternatives, which in turn would render inhibition more difficult and more time consuming, it is not unreasonable to conjecture that part of the increase in reaction time which has been found as a function of the number of alternatives is due to the increasing difficulty of the inhibitory processes associated with the incorrect responses.

Whether or not this conjecture is verified by further work, the adequacy of controlling for finger differences by imbedding the same finger in all the experimental conditions appears to have been brought into question by the present study.

\section{Referenees}

FITTS, P. M. Human information handling in speeded tasks. IBM Research Paper RC-109, 1959.

HICK, W. E. On the rate of gain of information. Quart. J. exp. Psychol., 1952, 4, 11-26.

RATZ, H. C., \& RITCHIE, D. K. Operator performance on a chord keyboard. J. appl. Psychol., 1961, 45, 303-308.

SEIBEL, R. Performance on a five-finger chord keyboard. IBM Research Paper RC-574, 1961.

\section{Noter}

1. This research was partly supported by an NIH Postdoctoral Fellowship, MF-12,990, which the author held at the Applied Psychology Research Unit in Cambridge, England; partial support also came from NIH grant MH-07197. The author wishes to express his appreciation to Drs. R. Conrad, and J.E.K. Smith for helpful comments.

2. The reaction time for the ipsilateral index finger was $303 \mathrm{~ms}$, for the contralateral index finger $287 \mathrm{~ms}$.; this $16 \mathrm{~ms}$. difference, in the same direction as the main result, is significant at beyond the .05 level $(t=2, d f=11)$. 